\title{
Long Non-coding RNA LINC02195 as a Regulator of MHC I Molecules and Favorable Prognostic Marker for Head and Neck Squamous Cell Carcinoma
}

\author{
Hao $\mathrm{Li}^{1+}$, Hong-Gang Xiong ${ }^{1 \dagger}$, Yao Xiao ${ }^{1}$, Qi-Chao Yang ${ }^{1}$, Shao-Chen Yang ${ }^{1}$, \\ Hong-Chao Tang ${ }^{1}$, Wen-Feng Zhang ${ }^{1,2 \star}$ and Zhi-Jun Sun ${ }^{1,2 \star}$ \\ ${ }^{1}$ The State Key Laboratory Breeding Base of Basic Science of Stomatology (Hubei-MOST) \& Key Laboratory of Oral \\ Biomedicine Ministry of Education, School \& Hospital of Stomatology, Wuhan University, Wuhan, China, ${ }^{2}$ Department of Oral \\ and Maxillofacial Head Neck Surgery, School \& Hospital of Stomatology, Wuhan University, Wuhan, China
}

OPEN ACCESS

Edited by:

Wei Cao,

Shanghai Jiao Tong University, China

Reviewed by:

Cheng Wang,

Sun Yat-sen University, China

Xuan Zhou,

Tianjin Medical University Cancer

Institute and Hospital, China

${ }^{*}$ Correspondence:

Wen-Feng Zhang

zhangw559@whu.edu.cn

Zhi-Jun Sun

sunzj@whu.edu.cn

†These authors have contributed equally to this work

Specialty section: This article was submitted to Head and Neck Cancer, a section of the journal

Frontiers in Oncology

Received: 05 March 2020 Accepted: 03 April 2020

Published: 06 May 2020

Citation:

$\mathrm{Li} H$, Xiong $\mathrm{H}-\mathrm{G}$, Xiao Y, Yang Q-C, Yang $S-C$, Tang $H-C$, Zhang $W-F$ and Sun Z-J (2020) Long Non-coding RNA LINC02195 as a Regulator of MHC I

Molecules and Favorable Prognostic Marker for Head and Neck Squamous Cell Carcinoma. Front. Oncol. 10:615. doi: 10.3389/fonc.2020.00615
The loss of major histocompatibility complex class I (MHC I) molecules is an important mechanism by which cancer cells escape immunosurveillance in head and neck squamous cell carcinoma (HNSCC). Several long non-coding RNAs (IncRNAs) have been implicated in immune response and regulation including antigen processing and presentation. However, few studies on IncRNAs regulating MHC I expression in HNSCC have been conducted. In this study, MHC I related IncRNAs were identified from the The Cancer Genome Atlas (TCGA) HNSCC database. One of the IncRNAs, long intergenic non-protein coding RNA 2195 (LINC02195), was found to be associated with genes encoding MHC I molecules and patient prognosis in the TCGA database. KEGG and GO analyses suggested that LINC02195 was closely related to antigen processing and presentation. QRT-PCR revealed high expression of LINC02195 in human HNSCC tissues and HNSCC cell lines compared with normal mucosal tissues. in situ hybridization of the HNSCC tissue microarray revealed a correlation between high LINC02195 expression and a favorable prognosis in our patient cohort. Silencing of LINC02195 decreased MHC I protein expression, as evidenced by western blotting. Multiplex immunochemistry was performed to reveal the positive correlation between high LINC02195 expression and an increased number of $\mathrm{CD}^{+}$and $\mathrm{CD}^{+}{ }^{+} \mathrm{T}$ cells in the tumor microenvironment. Based on our study, LINC02195 is a promising prognostic marker and a target for future therapeutic interventions.

Keywords: IncRNA, MHC, TCGA, tumor microenvironment, T cell

\section{INTRODUCTION}

Head and neck squamous cell carcinoma (HNSCC) accounts for 90\% of all cases of head and neck cancers, which causes $\sim 430,000$ deaths worldwide (1). HNSCC normally occurs in epithelial cells, including the mucosal lining of the upper airway and food passages (2). The general primary treatments for HNSCC include surgery, radiotherapy, chemotherapy and targeted therapy (3). In addition to these traditional treatments, immune checkpoint blockades such as anti-programmed cell death 1 (anti-PD-1) and anti-programmed 
cell death 1 ligand 1 (anti-PD-L1) antibodies have displayed great efficacy in the treatment of HNSCC $(3,4)$. However, the response rate to immune checkpoint blockades remains poor $(3,5)$. To increase the response rate, we should reveal the immunosuppressive mechanisms of tumors.

One of the roles of the innate and adaptive immune systems is to eliminate tumors before they are detectable. One of the mechanisms by which malignant cells escape immunosurveillance and elimination is the loss or downregulation of major histocompatibility complex class I (MHC I) molecules, limiting the recognition of tumor antigens by cytotoxic $\mathrm{CD}^{+} \mathrm{T}$ cells, which attack malignant cells (6). MHC I molecules are encoded by human leukocyte antigen class I (HLA I) genes mainly including HLA-A,HLA-B, and $H L A-C$, and are expressed by all somatic cells in humans (7). The deregulation of MHC I has been observed in various forms of cancer (8-10). Patients with lower levels of MHC I expression have poor survival for HNSCC (10). In addition, downregulation of MHC I in cancer cells was recently shown to be a mechanism underlying the immunotherapy resistance of cancer (11).

Long non-coding RNAs (lncRNAs) comprise various RNAs that are $<200$ nucleotides in length and have no proteincoding capacity. Several lncRNAs are abnormally expressed in various forms of cancer and are involved in the occurrence and development of tumors (12-14). With an increased understanding of lncRNAs, the important value of lncRNAs in treating tumors or predicting the prognosis of patients with tumors has been recognized (15). Notably, studies have recently been reported that some lncRNAs are identified as regulators of human immune system, particularly in antitumor immunity (16-18). A recent study discovered the association between lncRNAs and MHC I molecules. According to previous gene expression studies, the expression of the lncRNA HCP5 is associated with HLA-B expression (19). The lncRNA HOTAIR was recently shown to promote HLA$G$ expression in gastric cancer (20). However, few studies on lncRNAs regulating MHC I expression in HNSCC have been performed.

Here, several differentially expressed lncRNAs were identified by analyzing of The Cancer Genome Atlas (TCGA) database. Furthermore, we investigated a highly expressed lncRNA, long intergenic non-protein coding RNA 2195 (LINC02195, ENSG00000236481), which is closely associated with HLA-A, $-B$, and $-C$ expression. Next, we investigated the biological function of LINC02195 using bioinformatic analysis based on TCGA. A human tissue microarray (TMA) and in situ hybridization (ISH) were used to reveal the clinical role of LINC02195, and patients with high LINC02195 expression achieved a good outcome in the HNSCC patient cohort. As shown in western blots, LINC02195 silencing decreased the expression of MHC I molecules. By performing multiplex staining, a significant correlation between LINC02195 and CD8 ${ }^{+}$ and $\mathrm{CD}^{+} \mathrm{T}$ cell infiltration in the HNSCC microenvironment was revealed.

\section{MATERIALS AND METHODS}

Detailed information about the material and methods is provided in the Supplementary Material.

\section{Study Population, RNA Expression Data, and Bioinformatic Analysis}

The RNA expression data for HNSCC cases, which included 502 HNSCC tumor samples and 44 normal tissue samples were acquired from the TCGA database derived from the data portal (https://gdc.cancer.gov/). The dataset included the expression of RNA (mRNA and non-coding RNA) (level 3) and clinical data from 546 individuals. RNAs were identified using the Ensembl database. The differentially expressed lncRNAs (DElncRNAs) and mRNAs (DEmRNAs) were identified using the “edgeR" package. DEIncRNAs and DEmRNAs were analyzed by constructing a volcano plot with the "ggplot2" package in the $\mathrm{R}$ language.

\section{Gene Enrichment and Functional Annotation Analysis}

A subsequent functional enrichment analysis of the mRNAs ( $q$ values $\geq 0.4$ ) was performed. The bubble map was drawn using the "ggplot2" R package. The mRNAs with significant Pearson's correlation coefficient values (|Pearson's correlation coefficient $\mid \geq 0.4$ ) were included in further functional enrichment analyses. The Gene Ontology (GO) and Kyoto Encyclopedia of Genes and Genomes (KEGG) analyses were performed using the "clusterProfiler" package. The significant GO terms and KEGG pathways were identified as LINC02195-related biological functions and signaling pathways. Hierarchical clustering was performed using the "pheatmap" package in R language.

\section{Human HNSCC Samples and Analysis}

Ethical approval for this study was obtained from the Medical Ethics Committee of the School and Stomatology of Wuhan University (PI: Zhi-Jun Sun; 2014LUNSHENZI06). Human HNSCC samples were obtained from the Department of Oral and Maxillofacial Surgery, School and Hospital of Stomatology Wuhan University. The TNM classification at diagnosis was determined according to the 8th edition of TNM Classification of Malignant Tumors (UICC). The patient cohort included 5 patients with normal oral mucosae, 28 with oral epithelial dysplasia and 59 with HNSCC and 10 paired fresh HNSCC samples. The clinical characteristics, including TNM classification, histological grade and overall survival were available for all patients.

ISH was performed on a human HNSCC TMA with digoxigenin-labeled antisense oligonucleotide probes to examine the expression of LINC02195, as previously described (21). The probe sequence for LINC02195 was 5'-DIGTCCTTTGGAATCCTCCTACTTTGGCAGC-3'. IHC staining was performed as described (22). Signals were detected using biotinylated goat anti-rabbit or anti-mouse antibody followed by streptavidin HRP. Staining was visualized with DAB (Dako, USA), counterstained with hematoxylin (Dako), sealed with 
neutral resins, and imaged. The scanning of the TMA and processing of histoscores were performed using previously described methods (22). A human leukocyte antigen (HLA) class I ABC antibody (1:300, Proteintech, USA) was used to detect MHC I molecules in human HNSCC samples.

\section{Cell Lines, siRNAs, and Western Blotting}

The cell lines SCC4, SCC9, and CAL27 were obtained from ATCC (American Type Culture Collection) and maintained as previously described (23). TCA8113 cells were acquired from the Ninth People's Hospital, Shanghai Jiao Tong University and maintained in DMEM containing $1 \%$ penicillin and streptomycin (Thermo Fisher, USA) and 10\% fetal bovine serum (FBS, Gibco, USA). The human oral keratinocyte cell line (HOK) was obtained from ScienCell.

Small interfering RNAs (siRNAs) targeting LINC02195 were purchased from GenePharma (China). SCC9 cells seeded in a 6-well plate were transfected with the siRNAs using Lipofectamine 3,000 (Invitrogen, USA) according to the manufacturer's instructions.

Western blotting with whole-cell protein extracts from SCC9 cells was performed as previously described (21). An HLA class I ABC antibody (15240-1-AP; Proteintech, USA) was used for western blotting. GAPDH served as an internal loading control. All western blots were performed three times.

\section{Total RNA Extraction and Quantitative Reverse Transcription Polymerase Chain Reaction (qRT-PCR) Analysis}

The total RNA extraction protocol and qRT-PCR analysis have been described previously (21). LINC02195 expression was calculated with the comparative $\mathrm{Ct}$ method $\left(2^{-\Delta \Delta \mathrm{CT}}\right)$ and normalized to GAPDH expression. All qRT-PCR experiments were performed three times.

\section{Multiplex IHC and Image Analysis}

The Opal 7-Color Manual IHC Kit (NEL811001KT; PerkinElmer, Hopkinton, MA, USA) was used to stain the TMA as previously described (24). Briefly, for CD4, CD8, PD-1, and pan-keratin 4plex staining, 4 types of tyramide system amplification (TSA) sensors (Opal520, Opal570, Opal620, and Opal690) were used. After all multiplex TSA staining protocols was completed, the slide was stained with DAPI and mounted. All slides were scanned using the PerkinElmer Vectra system (PerkinElmer). The tumor and stromal area were distinguished and calculated with inForm (inForm 2.1.1; PerkinElmer) software using pankeratin as a tumor marker. From these data, the positive cell density was calculated using the following formula: positive cell density $=$ the number of positive cells in the stroma $\div$ the area of the stroma (calculating by image pixels).

\section{Statistical Analysis}

All data were statistically analyzed using Prism 8 software (GraphPad Software). One-way ANOVA ( $>2$ groups) or Student's $t$-tests ( 2 groups) ( $>2$ groups) were used to determine the significance of differences. Correlations were determined by calculating Pearson's correlation coefficient. Kaplan-Meier curves and Cox proportional hazards models were used to assess the prognostic value. Error bars are shown in the figures, and data are presented as the mean \pm SEM. $P<0.05$ was considered to be statistically significant, ${ }^{*} P<0.05,{ }^{* *} P<0.01$, and ${ }^{* * *} P<0.001$.

\section{RESULTS}

\section{LINC02195 Was Closely Correlated With HLA / Expression in the TCGA Database}

The TCGA database was used to investigate and analyze the expression of lncRNAs and mRNAs with the "edgeR" package. Notably, 5,731 upregulated DEmRNAs and DElncRNAs and 3,397 downregulated DEmRNAs and DElncRNAs were identified in HNSCC tissues (Figure 1A). Then, we extracted DElncRNA from the TCGA database and explored the correlations with the genes encoding MHC I molecules in humans (HLA$A$, $H L A-B$, and $H L A-C)$. The Venn diagram revealed eight DElncRNAs that were simultaneously associated with HLA$A$, HLA-B, and HLA-C, namely, PSMB8-AS1, TTLL11-IT1, LINC02195, LINC00623, LINC07871, LINC00944, LINC02574, and MIR3945HG (Figure 1B and Supplementary Figure 1A, |Pearson's correlation coefficient $\mid \geq 0.4$ ). According to the subsequent survival analysis, only LINC02195 and LINC01871 were correlated with patient prognosis (Figure 1C and Supplementary Figures 1B-H). As shown in Figure 1C, the Kaplan-Meier analysis revealed a significant correlation between high LINC02195 expression and good prognoses for patients with HNSCC in the TCGA database $(P=0.0180)$. The median histoscore was used as the cut-off. The Cox proportional hazards model also showed an association between LINC02195 expression and a good outcome (Table 1). Considering the correlation with the expression of MHC I molecules and prognostic value, LINC02195 was selected for further research in this study.

\section{LINC02195 Was Expressed at High Levels in HNSCC and Correlated With a Good Prognosis}

We next analyzed the expression and clinical features of LINC02195 in patients with HNSCC. LINC02195 was expressed at higher levels in tumor samples than in adjacent nontumor tissues (Figure 2A, $P<0.001$ ). Subsequently, 10 pairs of fresh HNSCC tissues matched with adjacent non-tumor tissues were used to verify the differential expression of LINC02195. Based on the qRT-PCR data, the expression in the HNSCC group was higher than that in the adjacent normal tissue group (Figure 2B, $P=0.0012$ ). Then we explored the expression of LINC02195 in human oral keratinocytes (HOKs) and the HNSCC cell lines SCC9, SCC4, TCA8113, and CAL27 using qRT-PCR. LINC02195 was expressed at significantly higher levels in HNSCC cell lines than in HOKs (Figure 2C). Using ISH combined with a tissue microarray, LINC02195 was expressed at higher levels in HNSCC tissues than in 

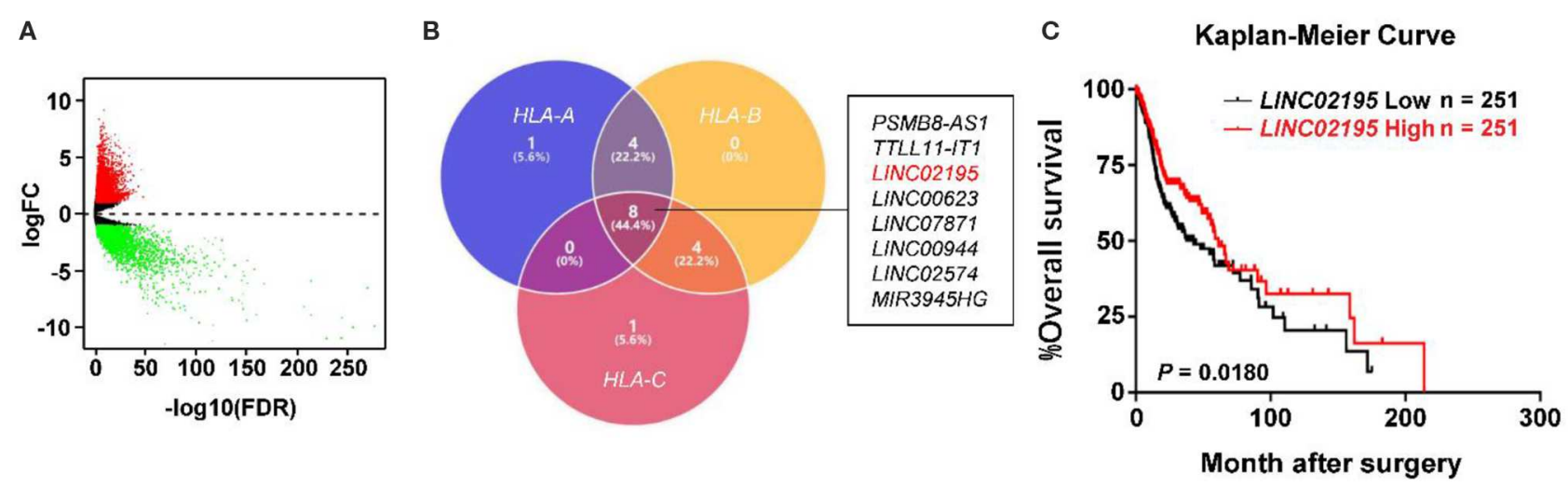

FIGURE 1 | LINC02195 expression was closely correlated with MHC I molecules in the TCGA database. (A) Volcano plot of the differentially expressed IncRNAs between HNSCC tissues and adjacent tissues. The log2(fold change) on the X axis and-log10(P-value) on the $Y$ axis are shown in the graph. Red dots represent high expression, and green dots represent low expression. Black shows IncRNA expression with both logFC $<1$ and - log10 ( $P$-value) $<0.05$. Differentially expressed mRNAs and IncRNAs were calculated using edgeR, which identified upregulated overexpressed IncRNAs and 3,397 downregulated IncRNAs. (B) Venn diagram showing that eight DEIncRNAs simultaneously associated with HLA-A, HLA-B, and HLA-C. (C) Kaplan-Meier survival curve of patients stratified according to low and high expression of LINC02195 in the TCGA database (HNSCC). The median expression was used as the cut-off; $P=0.0180$.

dysplastic tissue or the normal oral mucosa (Figures $2 \mathrm{D}, \mathrm{E}$, $P<0.001)$.

We analyzed the prognostic value of LINC02195 and the relationship between clinicopathological data and LINC02195 expression levels in the TCGA database and our patient cohort. The median histoscore was used as the cut-off. As shown in Figure 2F, the Kaplan-Meier analysis revealed a correlation between high LINC02195 expression and a good prognosis for patients included in the TMA $(P=0.0144)$, consistent the result observed in TCGA. The Cox proportional hazards model also showed an association between LINC02195 expression and a good outcome (Table 2).

As shown in Table 3, LINC02195 expression was not correlated with other clinical data in the TCGA database including age, sex, pathological grade, pathological stage, $\mathrm{T}$ classification, and $\mathrm{N}$ classification. One-way ANOVA and post-Tukey test of the human HNSCC tissue microarray data did not reveal associations of the LINC02195 expression level with the pathological grade, $\mathrm{T}$ classification, $\mathrm{N}$ classification, recurrence, radiotherapy, lymphatic metastasis, smoking, or alcohol consumption (Supplementary Figure 2).

\section{LINC02195 Was an Immune-Related IncRNA}

LINC02195-related mRNAs were selected for the gene annotation enrichment analysis to explore the biological functions of LINC02195. Sixty three enriched GO terms and 50 enriched KEGG pathways were associated with LINC02195 (Figure 3 and Supplementary Table 1,2, $P<0.05$ ). As shown in Figure 3A, the most significantly enriched GO terms were carbohydrate binding, receptor ligand activity, cytokine receptor binding and activity and cytokine binding and activity. Similarly, the significantly enriched pathways were cytokine-cytokine receptor interaction, cell adhesion molecules, antigen processing and presentation, hematopoietic cell lineage and Th1 and Th2 cell differentiation (Figures 3B,C). Interestingly, in terms of cytokine-cytokine receptor interactions and cell adhesion molecules, the GO analysis indicated that LINC02195 was closely correlated with immune factors such as chemokines, class I and II helical cytokines and the $\mathrm{T}$ cell receptor signaling pathway (Supplementary Figure 3). Based on these findings, LINC02195 was an immune-related lncRNA.

\section{LINC02195 Silencing Decreased the Expression of MHC I Molecules in HNSCC}

The GO enrichment analysis also revealed a close association of LINC02195 with MHC I protein binding (GO:0042288, $P=1.67 \mathrm{E}-12$, Supplementary Table 1). Moreover, KEGG pathway analyses identified close associations of LINC02195 with antigen processing and presentation (hsa04612, $P=3.00 \mathrm{E}-30$, Supplementary Table 2). As shown in Figure 4A, LINC02195 was closely correlated with the expression of $H L A-A, H L A-B$, and $H L A-C$, which encode classical MHC I molecules. Next, using an anti-HLA ABC antibody, the significant correlation between LINC02195 and MHC I was confirmed in our patient cohort (Figures 4B,C, $P<0.001$ ). In vitro, siLINC02195 was used to silence the expression of LINC02195, and HLA-A, -B, and -C expression was downregulated when LINC02195 was silenced (Figures 4D,E, $P<0.001$ ).

\section{LINC02195 Correlated With $\mathrm{CD}^{+}$and $\mathrm{CD}^{+}{ }^{+} \mathrm{T}$ Cell Infiltration in the HNSCC Tumor Microenvironment (TME)}

According to the KEGG pathway analysis, LINC02195 was closely correlated with genes that are important for antigen processing and presentation (Figure 5A). T cells in the TME, which detect the antigens presented by MHC molecules, have been 
A

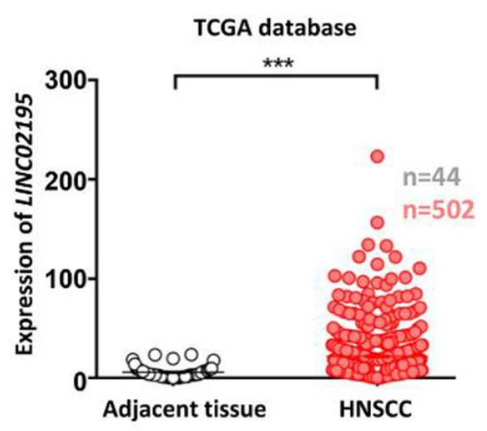

C

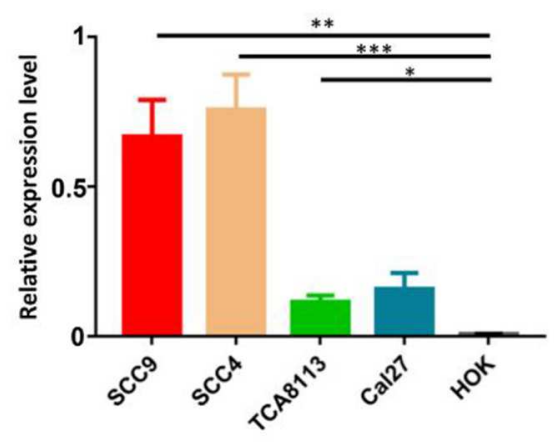

E

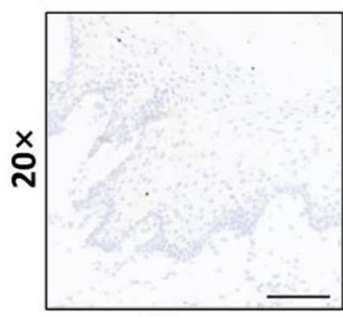

Mucosa

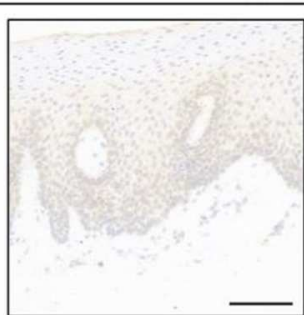

Dysplasia
B

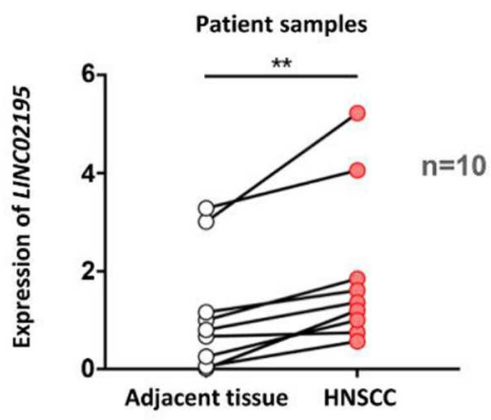

D

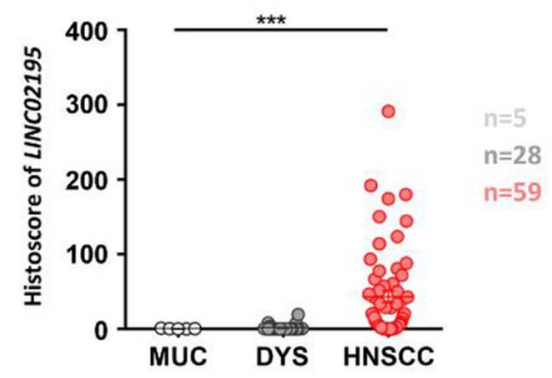

LINC02195

F Kaplan-Meier Curve

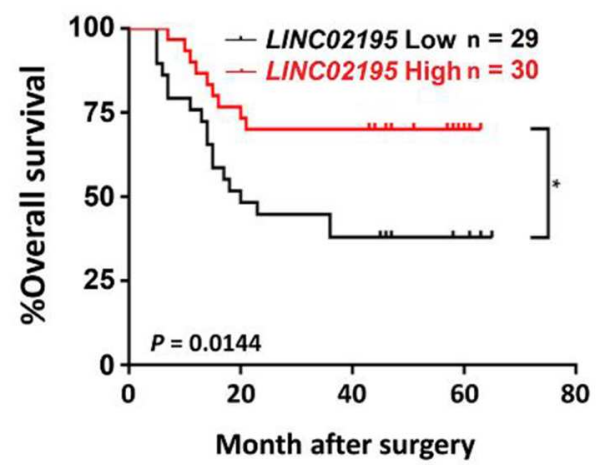

FIGURE 2 | LINC02195 was expressed at high levels in HNSCC tissues. (A) Relative expression level of LINC02195 in HNSCC and adjacent tissues from TCGA database. ${ }^{\star \star \star} P<0.001$. (B) Relative expression level of LINC02195 in HNSCC and adjacent tissue samples from patients with HNSCC. ${ }^{\star \star} P<0.01$. (C) Relative expression level of LINC02195 in 5 HNSCC cell lines (SCC9, SCC4, TCA8113, CAL27) and HOKs. ${ }^{\star} P<0.05$, ${ }^{\star *} P<0.01$, and ${ }^{* \star *} P<0.001$. (D-E) Statistical analysis (F) and representative images (E) of ISH using the probe for LINC02195 in normal mucosal, dysplastic and tumor tissues. Scale bar, $100 \mu \mathrm{m}$. (F) Kaplan-Meier curve of patients stratified according to low and high expression of LINC02195 in the cohort of the TMA. The median expression was used as the cut-off; $P=0.0144$. 
TABLE 1 | Multivariate analysis of the overall survival of patients with HNSCC based on TCGA.

\begin{tabular}{lcc}
\hline Parameters & HR (95\% & P-value \\
\hline Sex & $0.813(0.594-1.113)$ & 0.196 \\
Age & $1.021(1.007-7.035)$ & $0.003^{\star}$ \\
Pathological grade & $1.047(0.866-1.266)$ & 0.637 \\
Pathological stage & $1.136(0.964-1.338)$ & 0.127 \\
Tumor size & $1.025(0.901-1.164)$ & 0.711 \\
Node stage & $1.125(0.972-1.303)$ & 0.115 \\
LINC02195 expression & $0.743(.0557-0.991)$ & $0.043^{*}$
\end{tabular}

Cox proportional hazards regression model.

HR hazard ratio, 95\% Cl 95\% confidence interval.

${ }^{*} P<0.05$

TABLE 2 | Multivariate analysis of overall survival in patients with primary HNSCC.

\begin{tabular}{lcc}
\hline Parameters & HR (95\% Cl) & $P$-value \\
\hline Sex & $0.866(0.230-3.260)$ & 0.832 \\
Age & $2.278(0.905-5.731)$ & 0.080 \\
Pathological grade & $1.333(0.327-5.434)$ & 0.688 \\
Tumor size & $1.363(0.801-2.318)$ & 0.254 \\
Node stage & $1.287(0.767-2.162)$ & 0.339 \\
Smoking & $0.850(0.245-2.946)$ & 0.798 \\
Alcohol use & $1.046(0.349-3.134)$ & 0.935 \\
LINC02195 expression & $0.366(0.140-0.956)$ & $0.040^{*}$ \\
\hline
\end{tabular}

Cox proportional hazards regression model.

HR hazard ratio, 95\% Cl 95\% confidence interval.

${ }^{\star} P<0.05$

reported to be associated with good clinical outcomes in patients with many types of cancer, including bladder, breast, ovarian, colorectal, and renal cancers $(25,26)$. The correlation between MHC I and LINC02195 expression was previously proven. Additionally, the correlation between LINC02195 and MHC II molecules was also highly significant (Supplementary Figure 4). Therefore, the correlation between LINC02195 and T cells in TME was analyzed. By performing a bioinformatics analysis, we observed close correlations of LINC02195 expression with $C D 3 D, C D 3 E, C D 8 A, C D 8 B$, and CD4 expression in the TCGA database (Figures 5B,C). Pearson's correlation analysis revealed a strong association between LINC02195 and T cells in the TME. Based on the hierarchical clustering analysis, LINC02195 was more relevant to $\mathrm{CD}^{+} \mathrm{T}$ cells than to $\mathrm{CD} 4^{+}$ T cells.

Multiplex IHC was performed to analyze the immune status in the TME and verify this finding. As shown in Figure 6, tumor tissues with high LINC02195 expression exhibited more infiltrating $\mathrm{CD}^{+}$and $\mathrm{CD}^{+} \mathrm{T}$ cells than tumor tissues with low LINC02195 expression (Figure 6A). Interestingly, Pearson's correlation analysis identified a positive correlation between the increase in LINC02195 expression and the number of infiltrating $\mathrm{CD}^{+}{ }^{+}$and $\mathrm{CD} 4{ }^{+} \mathrm{T}$ cells (Figures 6B,C; CD8, $P<0.0001$; CD4, $P=0.0005)$. The hierarchical clustering analysis further clarified the relationship among these molecules (Figure 6D).
TABLE 3 | Relationship between LINC02195 expression and clinical parameters of patients in TCGA.

\begin{tabular}{|c|c|c|c|c|}
\hline \multirow[t]{2}{*}{ Clinical parameter } & \multirow[t]{2}{*}{$n$} & \multicolumn{3}{|c|}{ LINC02195 expression in TCGA database } \\
\hline & & Mean \pm SED & $t$ & $P$ \\
\hline \multicolumn{5}{|l|}{ Tumor vs. Normal } \\
\hline Adjacent non-tumor tissue & 44 & $5.68 \pm 0.9405$ & 4.123 & $<0.0001^{*}$ \\
\hline Tumor & 502 & $21.66 \pm 1.143$ & & \\
\hline \multicolumn{5}{|l|}{ Age (years) } \\
\hline$<60$ & 245 & $21.73 \pm 1.782$ & 0.1238 & 0.9016 \\
\hline$\geq 60$ & 255 & $21.44 \pm 1.458$ & & \\
\hline \multicolumn{5}{|l|}{ Sex } \\
\hline Male & 367 & $21.81 \pm 1.354$ & 0.2262 & 0.8211 \\
\hline Female & 134 & $21.22 \pm 2.149$ & & \\
\hline \multicolumn{5}{|l|}{ Pathological grade } \\
\hline $\mathrm{G} 1+\mathrm{G} 2$ & 362 & $20.48 \pm 1.3$ & 1.402 & 0.1616 \\
\hline $\mathrm{G} 3+\mathrm{G} 4$ & 120 & $24.12 \pm 2.234$ & & \\
\hline \multicolumn{5}{|l|}{ Pathological stage } \\
\hline Stage I-II & 95 & $22.93 \pm 2.487$ & 0.7006 & 0.4840 \\
\hline Stage III-IV & 338 & $20.89 \pm 1.377$ & & \\
\hline \multicolumn{5}{|l|}{$\mathrm{T}$} \\
\hline $\mathrm{T} 1+\mathrm{T} 2$ & 178 & $22.58 \pm 1.823$ & 0.9838 & 0.3258 \\
\hline $\mathrm{T} 2+\mathrm{T} 3$ & 267 & $20.22 \pm 1.538$ & & \\
\hline \multicolumn{5}{|l|}{$\mathrm{N}$} \\
\hline NO & 170 & $21.13 \pm 1.793$ & 0.2114 & 0.8327 \\
\hline $\mathrm{N} 1+\mathrm{N} 2+\mathrm{N} 3$ & 238 & $21.66 \pm 1.706$ & & \\
\hline
\end{tabular}

\section{DISCUSSION}

In this study, the expression of the lncRNA LINC02195 was closely associated with MHC molecules. Moreover, it was expressed at high levels in human HNSCC tissues, based on the analyses of TCGA database and patient samples. In addition, patients with high LINC02195 expression exhibited better prognoses than patients with low LINC02195 expression. We conducted GO and KEGG enrichment analyses to clarify the function and protein-protein interaction network of LINC02195 by identifying LINC02195-related mRNAs in HNSCC tissues in the TCGA database and to determine the explanation for this outcome. LINC02195 expression was closely correlated with the expression of MHC I molecules. Through further western blotting and immunostaining analysis, the correlation was further verified in vitro. Multiplex staining and Pearson's correlation analyses were used to verify the significant relationship between the lncRNA and infiltrating $\mathrm{CD}^{+}$and $\mathrm{CD}^{+} \mathrm{T}$ cells in TME.

LncRNAs have been widely investigated in recent years. An increasing number of lncRNAs have been shown to regulate the immune system (27). LINC02195, a newly annotated lncRNA, is located on chromosome 16p12.1. In normal tissues, it is expressed at high levels in the appendix, esophagus, lymph node, stomach, thyroid, some immune cell-rich places in our body, which suggest it may correlate with immune function (28). However, the relationship between LINC02195 and HNSCC has 
A

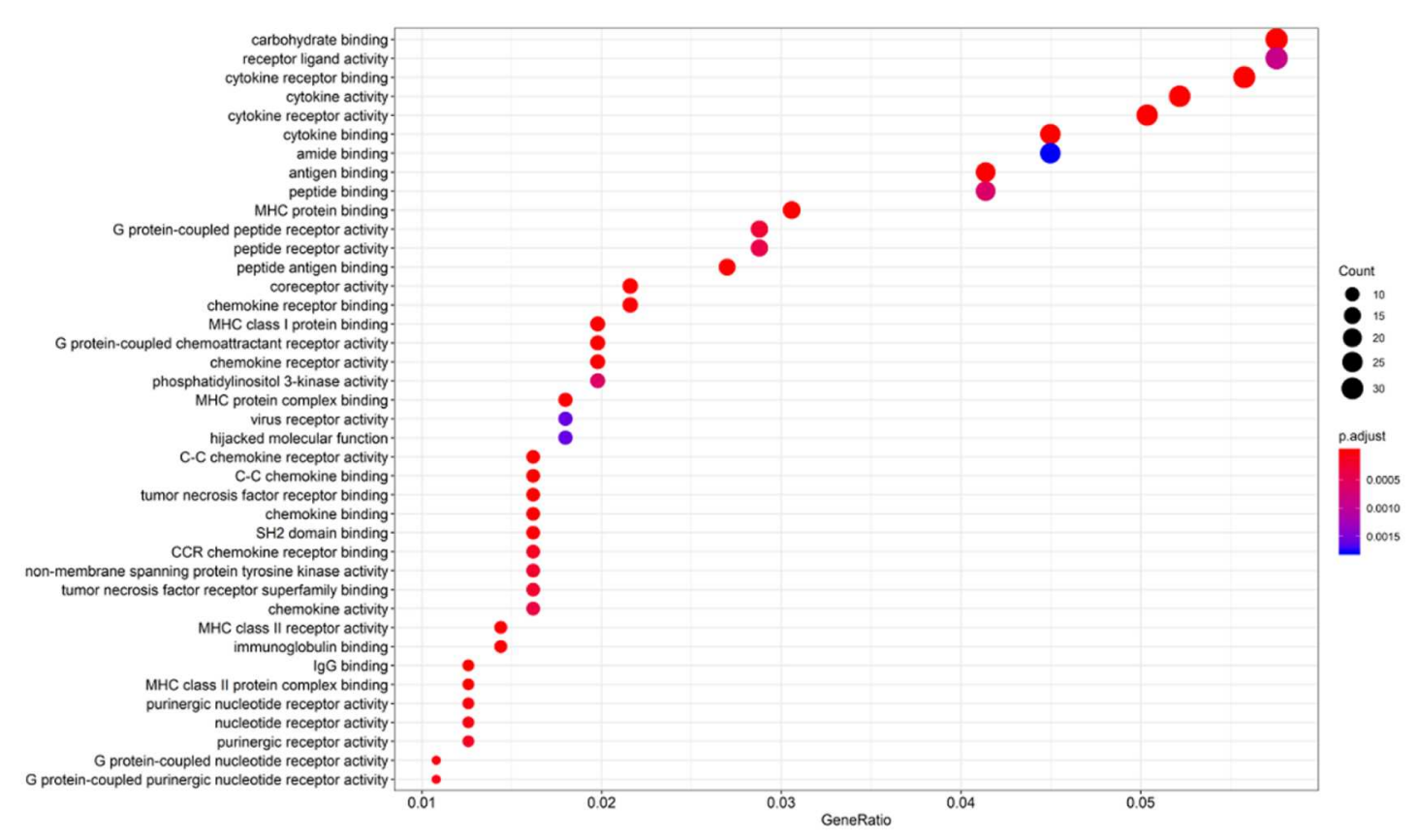

B

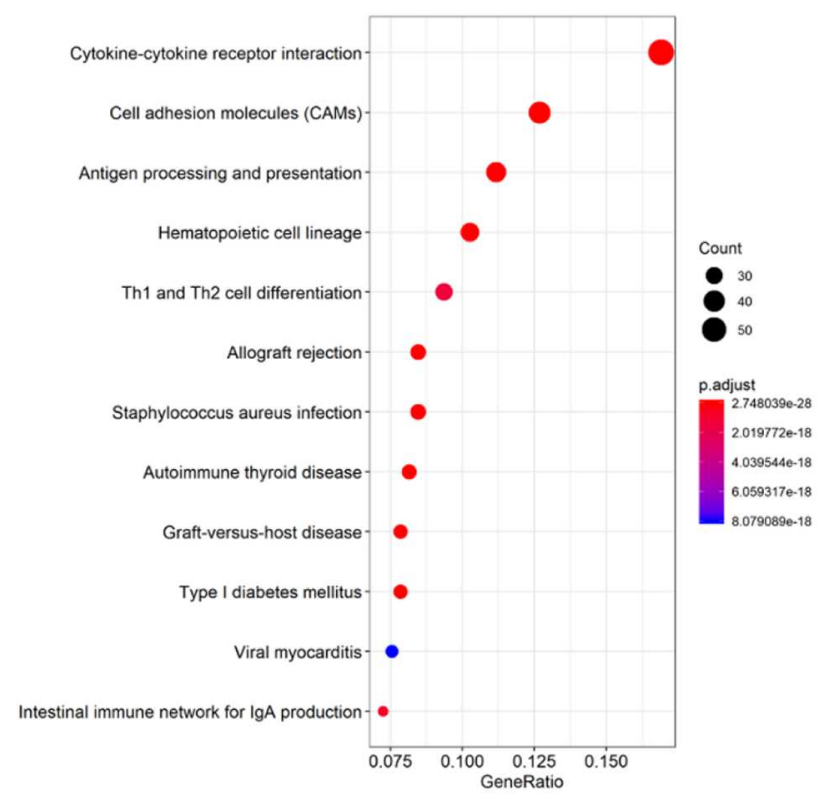

c

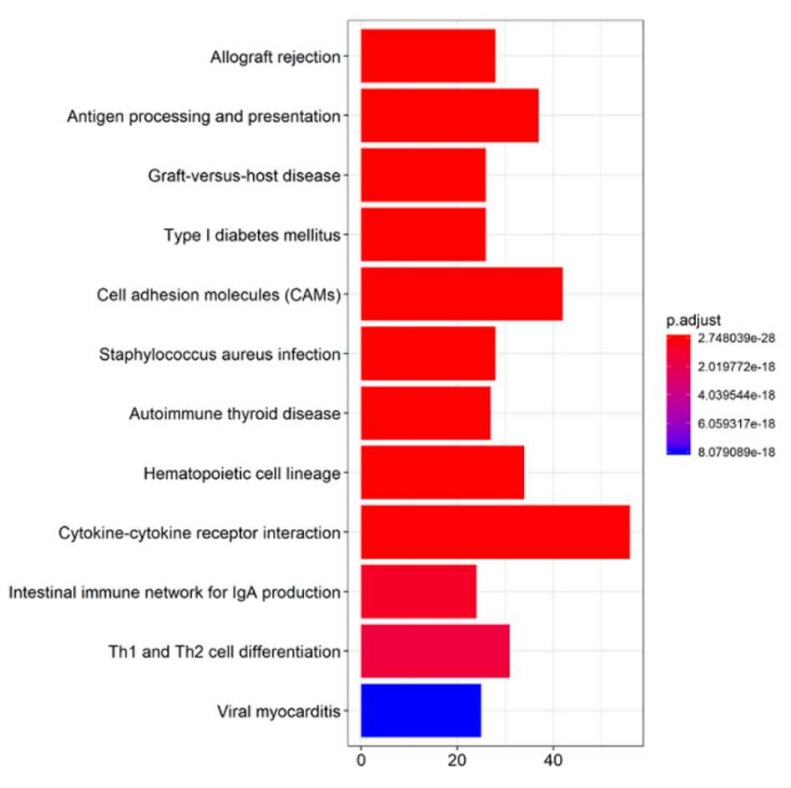

FIGURE 3 | GO and KEGG pathway enrichment analysis for LINC02195-related mRNAs based on TCGA database. (A) Plot of the enriched GO terms associated with LINC02195-related mRNAs. (B,C) Plot of the KEGG pathways associated with LINC02195-related mRNAs. $P<0.05$ and Cor $>0.4$ were used as the thresholds to select GO and KEGG terms.

not been revealed. In this study, LINC02195 was confirmed to be associated with HNSCC by analyzing HNSCC and normal tissues. We found LINC02195 is mostly found in the nucleus and cytoplasm by ISH. Moreover, high expression of this lncRNA was associated with a good prognosis for patients with HNSCC. GO and KEGG analyses revealed that LINC02195 was an immunerelated lncRNA. This lncRNA was correlated with antigen processing and presentation. MHC I molecules have a crucial role in this process, and previous studies have reported an association between the expression of the lncRNA HCP5 expression was associated with $H L A-B$ expression (19). In the present study, the Pearson's correlation analysis indicated a close correlation between genes encoding MHC I molecules with LINC02195. Moreover, western blotting revealed a decrease in MHC I levels 
A

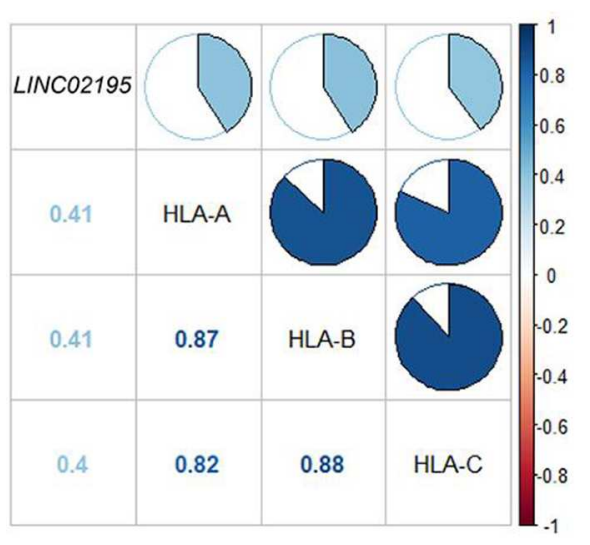

C

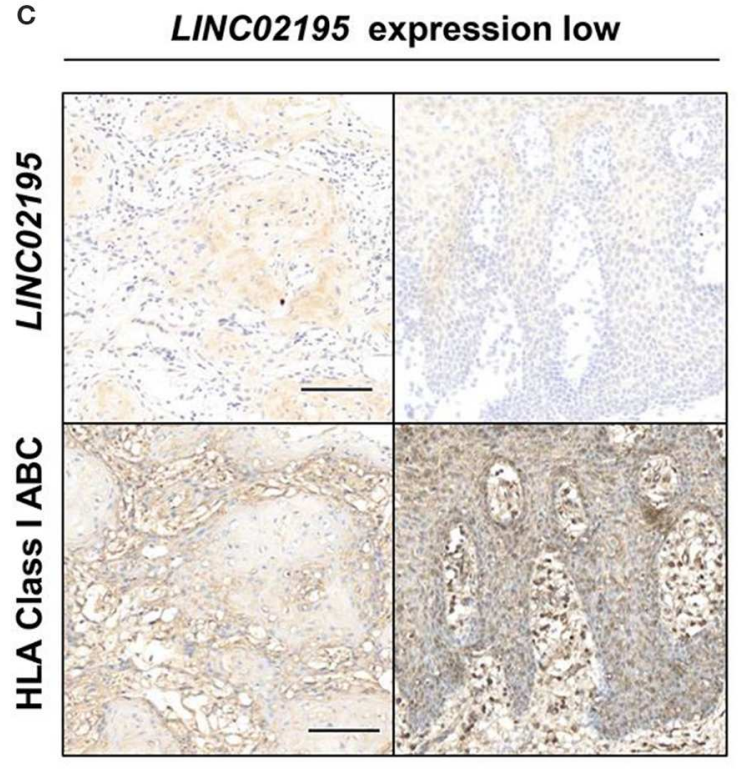

D

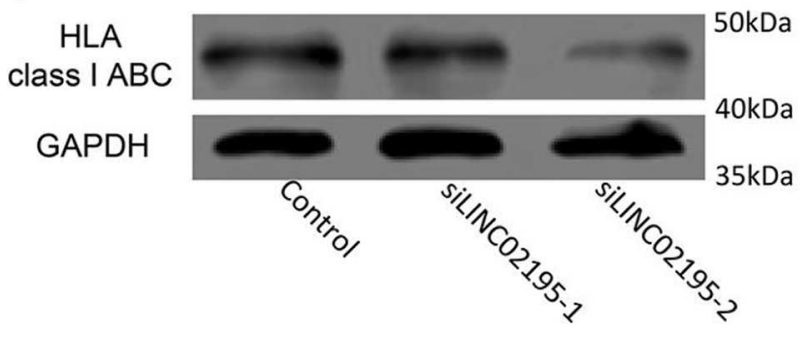

B

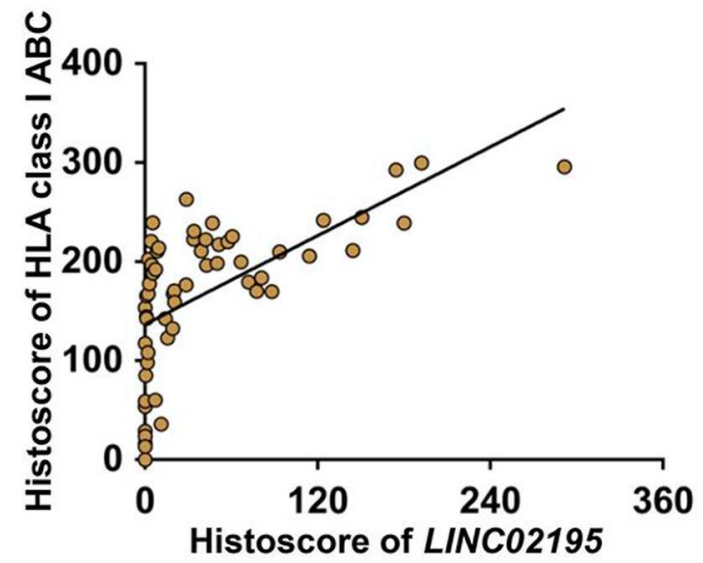

LINC02195 expression high

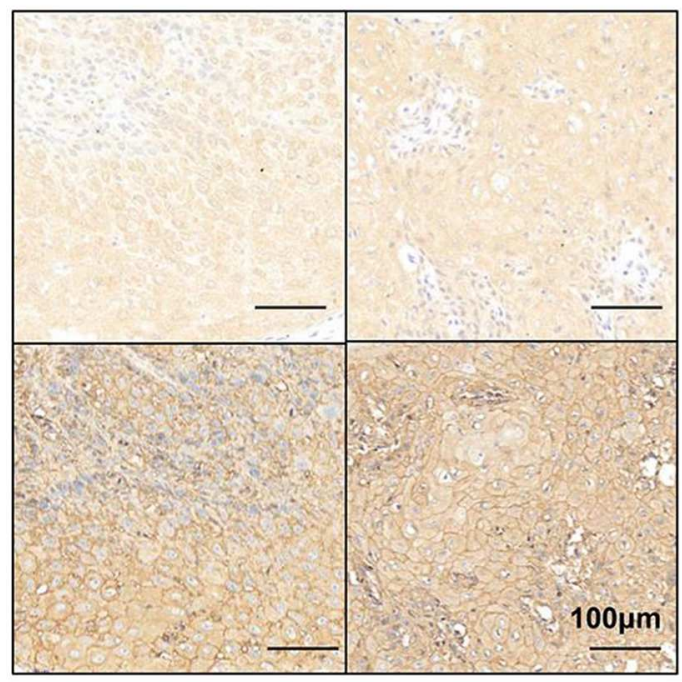

$E$

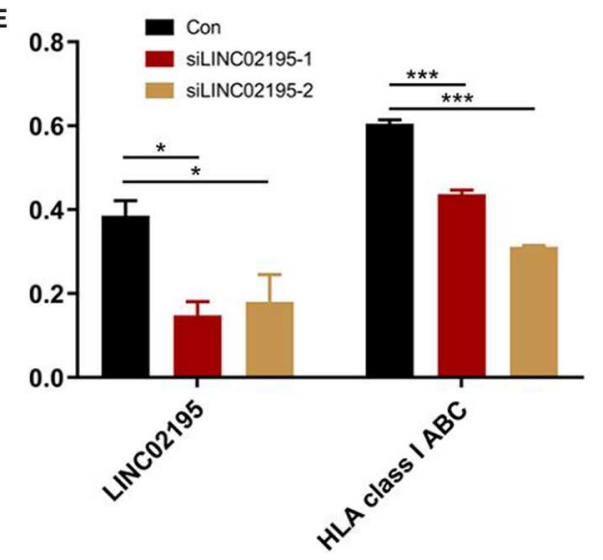

FIGURE 4 | LINC02195 was closely correlated with the expression of MHC I molecule in tumor cells. (A) Pearson's correlation coefficients among LINC02195, HLA-A, HLA-B, and HLA-C expression in HNSCC samples from TCGA database. (B) Pearson's correlation coefficients between the histoscore of $L I N C 02195$ and HLA class I ABC in the human HNSCC TMA; $P<0.0001, r=0.6103$. (C) Representative images of ISH and IHC for LINC02195 and HLA class I ABC in human HNSCC specimens (low LINC02195 expression vs. high LIN02195 expression). Scale bar: $100 \mu \mathrm{m}$. (D,E) in vitro, LINC02195 knockdown by using siLINC02195 downregulated the expression of HLA class I ABC in the SCC9 cell line. ${ }^{*} P<0.05$ and ${ }^{\star \star *} P<0.001$. 
A

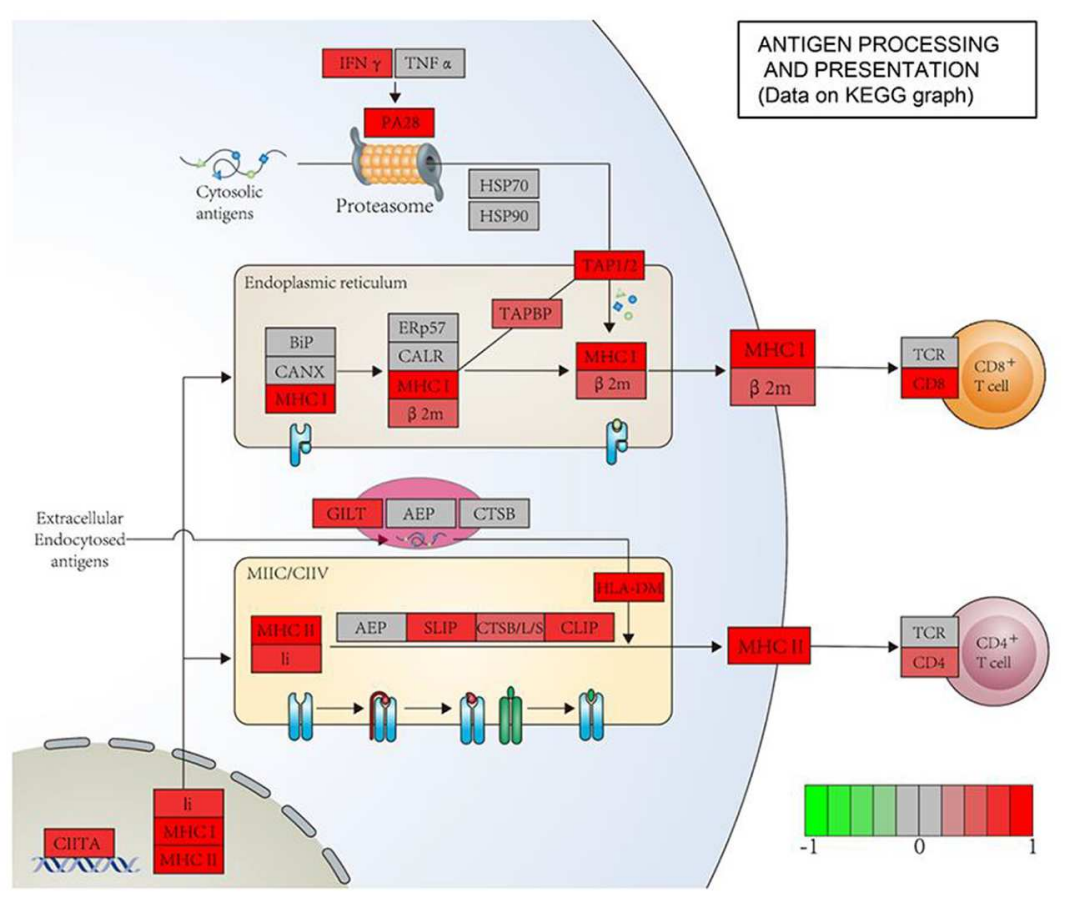

B
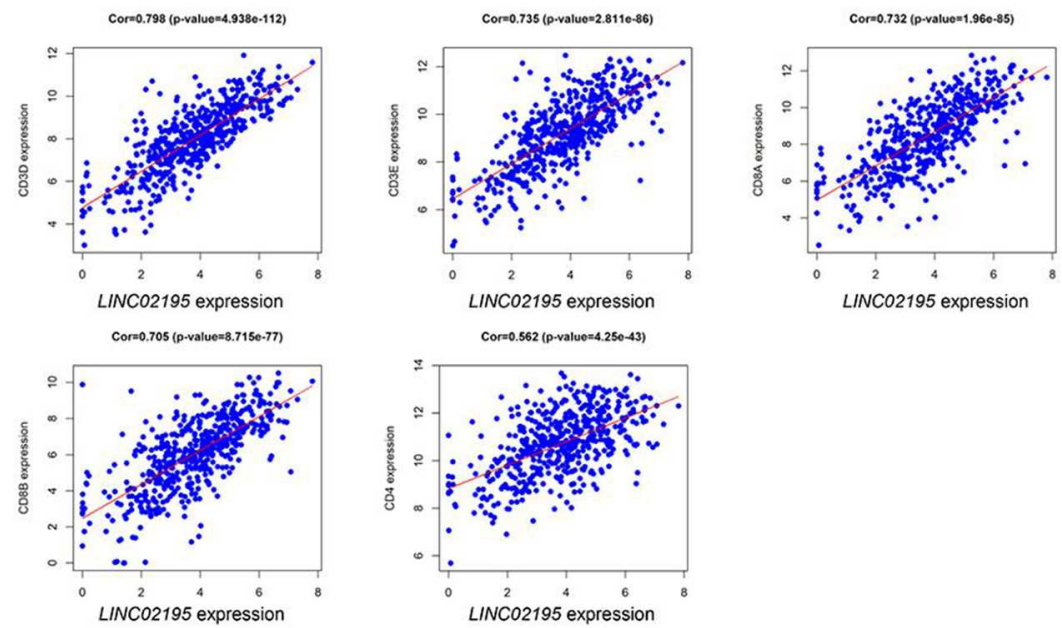

C

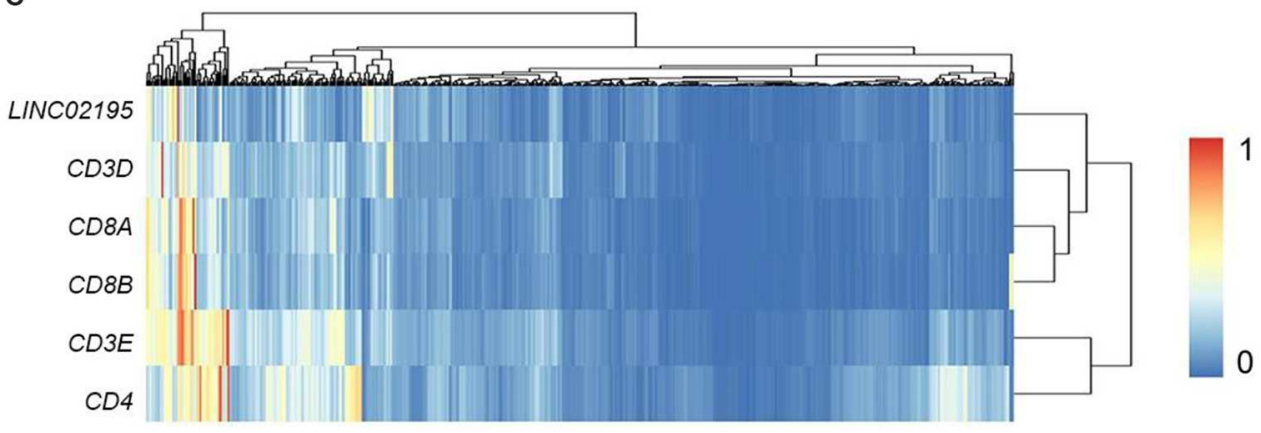

FIGURE 5 | LINC02195 expression was positively correlated with genes involved in antigen processing and presentation. (A) KEGG pathway enrichment analyses showed a close correlation between LINC02195 expression and antigen processing and presentation in both the MHC I and II pathways. (B) Pearson's correlation coefficients between LINC02195 and CD3D, CD3E, CD8A, CD8B, or CD4 gene expression based on TCGA data. (C) Hierarchical clustering analysis of LINC02195, $C D 3 D, C D 3 E, C D 8 A, C D 8 B$, and $C D 4$ in TCGA database. 
A
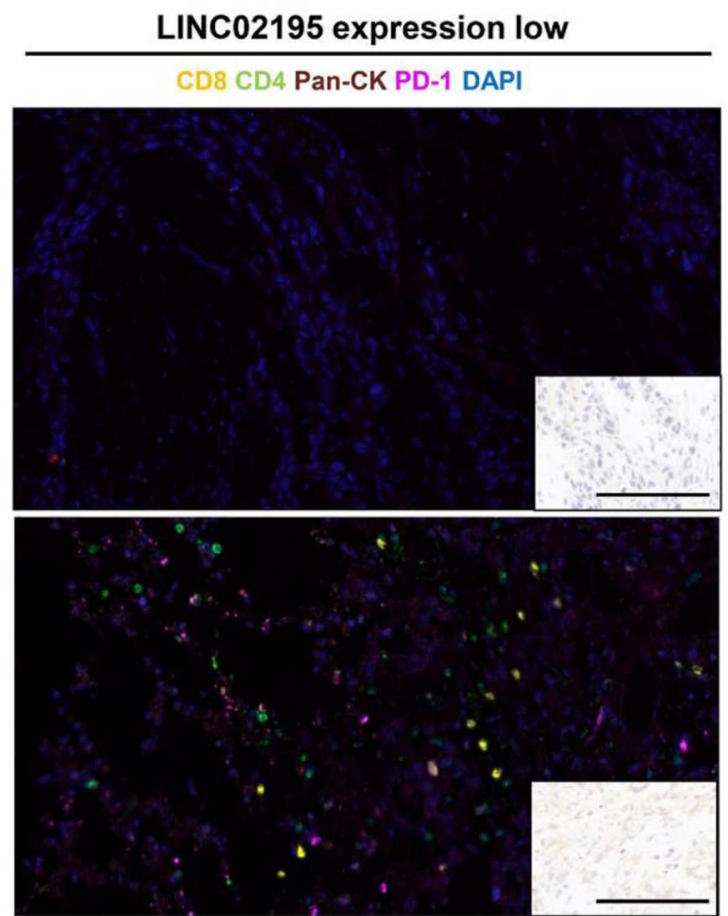

B

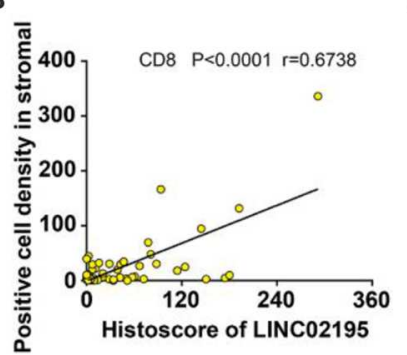

C

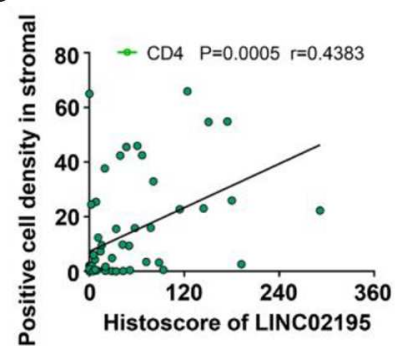

LINC02195 expression high

CD8 CD4 Pan-CK PD-1 DAPI
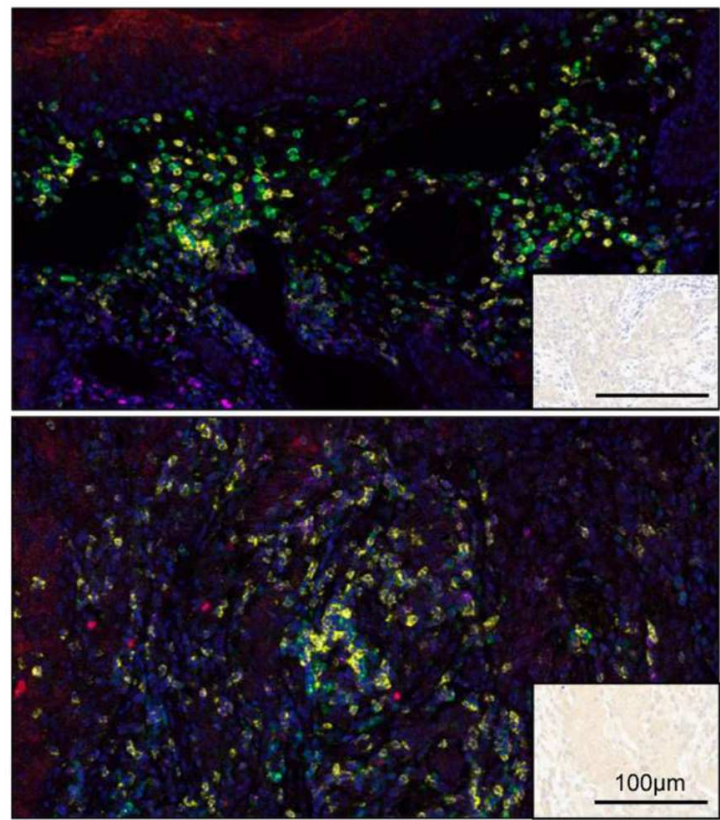

D

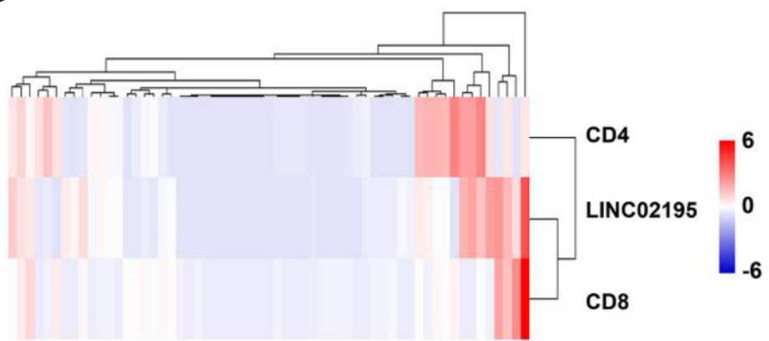

FIGURE 6 | LINC02195 expression was positively correlated with the infiltration of CD8 ${ }^{+}$and CD4 ${ }^{+}$T cells in the TME. (A) Representative image of multiplex staining (CD8, CD4, PD-1, pan-keratin, and DAPI) in human HNSCC specimens (low LINC02195 expression vs. high LINC02195 expression). Scale bar, 100 $\mu$ m. (B,C) Pearson's correlation coefficient between the histoscore of LINC02195 and the number of CD8 ${ }^{+}$or CD4 ${ }^{+}$cells in the human HNSCC TMA. (D) Hierarchical clustering analysis of LINC02195, CD4, and CD8 expression in human HNSCC samples $(n=59)$.

upon the silencing of LINC02195. The results suggested that LINC02195 may interact with related enzymes or molecules encoding MHC I in the nucleus and further regulating the expression of MHC I molecules.

In recent years, the TME has attracted increasing attention from researchers because of its close correlation with prognosis and treatment. A number of various types of immune cells infiltrate the TME (25). Substantial $\mathrm{T}$ cell infiltration is associated with good clinical outcomes and positive responses to immunotherapy in many types of cancer $(25,29)$. However, the relationship between lncRNAs and $\mathrm{T}$ cells in the TME remains unclear. As shown in a previous study, $\operatorname{lnc}-E G F R$ stimulates Treg differentiation and subsequently promotes the immune evasion of hepatocellular cancer (18). According to another study, a lncRNA, LINK-A, downregulated cancer cell antigen presentation and was negatively correlated with $\mathrm{T}$ cells in the TME of triple-negative breast cancer (30). In the present study, gene enrichment and pathway analyses identified a strong correlation between LINC02195 and T cells in the tumor context. Peptide-MHC I complexes are transported to the plasma membrane for cancer cell antigen presentation to $\mathrm{CD}^{+} \mathrm{T}$ cells, which attack malignant cells. Next, through multiplex IHC of the human HNSCC TMA, we observed a positive correlation between LINC02195 expression and the density of $\mathrm{CD}^{+}$and $\mathrm{CD}^{+}{ }^{+} \mathrm{T}$ cells in the TME.

In summary, this study is the first to explore the role of LINC02195 in HNSCC. High LINC02195 expression were correlated with a good prognosis for patients with HNSCC. LINC02195 may play a fundamental role in regulating the expression of MHC I molecules. In addition, LINC02195 was 
an immune-related lncRNA and positively correlated with an increased $\mathrm{T}$ cell density. This evidence, therefore, supports the hypothesis that LINC02195 is a promising prognostic marker for HNSCC and a target for future therapeutic interventions.

\section{DATA AVAILABILITY STATEMENT}

The raw data supporting the conclusions of this article will be made available by the authors, without undue reservation, to any qualified researcher.

\section{AUTHOR CONTRIBUTIONS}

HL and H-GX contributed to conception, design, data acquisition, analysis, drafted, and critically revised the manuscript. YX, Q-CY, S-CY, and H-CT contributed to data acquisition, drafted and critically revised the manuscript.

\section{REFERENCES}

1. Siegel RL, Miller KD, Jemal A. Cancer statistics, 2020. CA Cancer J Clin. (2020) 70:7-30. doi: 10.3322/caac. 21590

2. Wyss A, Hashibe M, Chuang SC, Lee YC, Zhang ZF, Yu GP, et al. Cigarette, cigar, and pipe smoking and the risk of head and neck cancers: pooled analysis in the International Head and neck cancer epidemiology consortium. Am J Epidemiol. (2013) 178:679-90. doi: 10.1093/aje/kwt029

3. Cramer JD, Burtness B, Le QT, Ferris RL. The changing therapeutic landscape of head and neck cancer. Nat Rev Clin Oncol. (2019) 16:66983. doi: 10.1038/s41571-019-0227-z

4. Ferris RL, Blumenschein G Jr, Fayette J, Guigay J, Colevas AD, Licitra L, et al. Nivolumab for recurrent squamous-cell carcinoma of the head and neck. $N$ Engl J Med. (2016) 375:1856-67. doi: 10.1056/NEJMoa1602252

5. Ferris RL. Immunology and immunotherapy of head and neck cancer. J Clin Oncol. (2015) 33:3293-304. doi: 10.1200/JCO.2015.61.1509

6. Leone P, Shin EC, Perosa F, Vacca A, Dammacco F, Racanelli V. MHC class I antigen processing and presenting machinery: organization, function, and defects in tumor cells. J Natl Cancer Inst. (2013) 105:117287. doi: 10.1093/jnci/djt184

7. Neefjes J, Jongsma ML, Paul P, Bakke O. Towards a systems understanding of MHC class I and MHC class II antigen presentation. Nat Rev Immunol. (2011) 11:823-36. doi: 10.1038/nri3084

8. Garrido F, Ruiz-Cabello F, Aptsiauri N. Rejection versus escape: the tumor MHC dilemma. Cancer Immunol Immunother. (2017) 66:25971. doi: 10.1007/s00262-016-1947-x

9. Aptsiauri N, Ruiz-Cabello F, Garrido F. The transition from HLA-I positive to HLA-I negative primary tumors: the road to escape from T-cell responses. Curr Opin Immunol. (2018) 51:123-32. doi: 10.1016/j.coi.2018.03.006

10. Yoo SH, Keam B, Ock CY, Kim S, Han B, Kim JW, et al. Prognostic value of the association between MHC class I downregulation and PD-L1 upregulation in head and neck squamous cell carcinoma patients. Sci Rep. (2019) 9:7680. doi: 10.1038/s41598-019-44206-2

11. Paulson KG, Voillet V, McAfee MS, Hunter DS, Wagener FD, Perdicchio $\mathrm{M}$, et al. Acquired cancer resistance to combination immunotherapy from transcriptional loss of class I HLA. Nat Commun. (2018) 9:3868. doi: 10.1038/s41467-018-06300-3

12. Prensner JR, Chinnaiyan AM. The emergence of lncRNAs in cancer biology. Cancer Discov. (2011) 1:391-407. doi: 10.1158/2159-8290.CD-11-0209

13. Xiu DH, Liu GF, Yu SN, Li LY, Zhao GQ, Liu L, et al. Long non-coding RNA LINC00968 attenuates drug resistance of breast cancer cells through inhibiting the Wnt2/beta-catenin signaling pathway by regulating WNT2. J Exp Clin Cancer Res. (2019) 38:94. doi: 10.1186/s13046-019-1100-8
W-FZ and Z-JS contributed to conception, data analysis, and interpretation, drafted and critically revised the manuscript. All authors provided final approval and agreed to be accountable for all aspects of the study.

\section{FUNDING}

This study was supported by grants from the National Natural Science Foundation of China 81874131, 81672668, and 81672667. Z-JS was supported by the Fundamental Research Funds for the Central Universities of China 2042017kf0171 (Outstanding Young Scholars).

\section{SUPPLEMENTARY MATERIAL}

The Supplementary Material for this article can be found online at: https://www.frontiersin.org/articles/10.3389/fonc. 2020.00615/full\#supplementary-material

14. Liu $\mathrm{H}$, Deng $\mathrm{H}$, Zhao $\mathrm{Y}$, Li $\mathrm{C}$, Liang $\mathrm{Y}$. LncRNA XIST/miR-34a axis modulates the cell proliferation and tumor growth of thyroid cancer through MET-PI3K-AKT signaling. J Exp Clin Cancer Res. (2018) 37:279. doi: 10.1186/s13046-018-0950-9

15. Wapinski O, Chang HY. Long noncoding RNAs and human disease. Trends Cell Biol. (2011) 21:354-61. doi: 10.1016/j.tcb.2011.04.001

16. Trimarchi $T$, Bilal E, Ntziachristos $P$, Fabbri G, Dalla-Favera $R$, Tsirigos A, et al. Genome-wide mapping and characterization of Notch-regulated long noncoding RNAs in acute leukemia. Cell. (2014) 158:593-606. doi: 10.1016/j.cell.2014.05.049

17. Wang P, Xue Y, Han Y, Lin L, Wu C, Xu S, et al. The STAT3-binding long noncoding RNA lnc-DC controls human dendritic cell differentiation. Science. (2014) 344:310-3. doi: 10.1126/science.1251456

18. Jiang R, Tang J, Chen Y, Deng L, Ji J, Xie Y, et al. The long noncoding RNA lnc-EGFR stimulates T-regulatory cells differentiation thus promoting hepatocellular carcinoma immune evasion. Nat Commun. (2017) 8:15129. doi: 10.1038/ncomms15129

19. Kulski JK. Long Noncoding RNA HCP5, a Hybrid HLA Class I endogenous retroviral gene: structure, expression, and disease associations. Cells. (2019) 8:480. doi: $10.3390 /$ cells 8050480

20. Song B, Guan Z, Liu F, Sun D, Wang K, Qu H. Long non-coding RNA HOTAIR promotes HLA-G expression via inhibiting miR-152 in gastric cancer cells. Biochem Biophys Res Commun. (2015) 464:80713. doi: 10.1016/j.bbrc.2015.07.040

21. Xiong HG, Li H, Xiao Y, Yang QC, Yang LL, Chen L, et al. Long noncoding RNA MYOSLID promotes invasion and metastasis by modulating the partial epithelial-mesenchymal transition program in head and neck squamous cell carcinoma. J Exp Clin Cancer Res. (2019) 38:278. doi: 10.1186/s13046-019-1254-4

22. Li H, Yang LL, Xiao Y, Deng WW, Chen L, Wu L, et al. Overexpression of Golgi Phosphoprotein 2 is associated with poor prognosis in oral squamous cell carcinoma. Am J Clin Pathol. (2018) 150:74-83. doi: 10.1093/ajcp/aqy029

23. Chen L, Yang QC, Li YC, Yang LL, Liu JF, Li H, et al. Targeting CMTM6 suppresses stem cell-like properties and enhances antitumor immunity in head and neck squamous cell carcinoma. Cancer Immunol Res. (2020) 8:17991. doi: 10.1158/2326-6066.CIR-19-0394

24. Xiao Y, Li H, Mao L, Yang QC, Fu LQ, Wu CC, et al. CD103(+) $\mathrm{T}$ and dendritic cells indicate a favorable prognosis in oral cancer. J Dent Res. (2019) 98:1480-7. doi: 10.1177/00220345198 82618

25. Fridman WH, Zitvogel L, Sautes-Fridman C, Kroemer G. The immune contexture in cancer prognosis and treatment. Nat Rev Clin Oncol. (2017) 14:717-34. doi: 10.1038/nrclinonc.2017.101 
26. Fridman WH, Pages F, Sautes-Fridman C, Galon J. The immune contexture in human tumours: impact on clinical outcome. Nat Rev Cancer. (2012) 12:298-306. doi: 10.1038/nrc3245

27. Li Y, Jiang $\mathrm{T}$, Zhou $\mathrm{W}$, Li J, Li X, Wang Q, et al. Pan-cancer characterization of immune-related lncRNAs identifies potential oncogenic biomarkers. Nat Commun. (2020) 11:1000. doi: 10.1038/s41467-02014802-2

28. Fagerberg L, Hallstrom BM, Oksvold P, Kampf C, Djureinovic D, Odeberg $\mathrm{J}$, et al. Analysis of the human tissue-specific expression by genomewide integration of transcriptomics and antibody-based proteomics. Mol Cell Proteomics. (2014) 13:397-406. doi: 10.1074/mcp.M113. 035600

29. Matsushita H, Vesely MD, Koboldt DC, Rickert CG, Uppaluri R, Magrini VJ, et al. Cancer exome analysis reveals a T-cell-dependent mechanism of cancer immunoediting. Nature. (2012) 482:400-4. doi: 10.1038/ nature 10755
30. Hu Q, Ye Y, Chan LC, Li Y, Liang K, Lin A, et al. Oncogenic lncRNA downregulates cancer cell antigen presentation and intrinsic tumor suppression. Nat Immunol. (2019) 20:83551. doi: 10.1038/s41590-019-0400-7

Conflict of Interest: The authors declare that the research was conducted in the absence of any commercial or financial relationships that could be construed as a potential conflict of interest.

Copyright (c) $2020 \mathrm{Li}$, Xiong, Xiao, Yang, Yang, Tang, Zhang and Sun. This is an open-access article distributed under the terms of the Creative Commons Attribution License (CC BY). The use, distribution or reproduction in other forums is permitted, provided the original author(s) and the copyright owner(s) are credited and that the original publication in this journal is cited, in accordance with accepted academic practice. No use, distribution or reproduction is permitted which does not comply with these terms. 